\title{
Healthy adjustment for new residents with dementia using SettleIN: a feasibility study in UK care homes.
}

\begin{abstract}
Objectives: This study aimed to develop and explore feasibility of SettleIN, a staff led programme about healthy adjustment for people with dementia following care home placement. The main foci were intervention feasibility and the impact of the programme on resident quality of life and mood.
\end{abstract}

Method: A manualised intervention developed through consultation with 47 experts was trialled using a mixed-method design. Thirteen new residents with dementia and 24 staff were recruited from six UK care homes. Outcomes were measured at baseline, intervention completion and four-week follow up. Analysis of staff interviews and field notes are reported.

Results: Most experts deemed SettleIN to be well-structured, comprehensive and appropriate. However, uptake of SettleIN was low. When implemented, staff emphasised integration ease and staff benefits, but that SettleIN may not be universally suitable. High attrition, most commonly due to death and hospitalisation; and partial results from only four participants meant there was a lack of support for the positive outcomes. Feasibility problems included a lack of staff time and dependency on families for some components. Conclusion: SettleIN is acceptable to a wide range of stakeholders though does not appear to be feasible in its current form and improvements are recommended. A second pilot phase is required, which will address the reasons for the high attrition rate in this study and amend the methodology accordingly. This is important work as a manualised and standardised approach to healthy adjustment in care is unique and could have huge clinical significance if effective. 


\section{Introduction}

Of the 46.8 million people worldwide living with dementia (Alzheimer's Disease International [ADI], 2015) one third to one half in high income countries are cared for in long-term care facilities (Prince et al., 2013). The number of people with dementia in England living in care homes is estimated to rise to 390,000 in 2031 (Comas-Herrera et al., 2011). Initial adjustment is a key stage in relocation to a care home (Smith \& Crome, 2000) and can take between two to four weeks or as long as six months (Ellis, 2010; Hodgson, Freedman, Granger, \& Erno, 2004).

Positive and negative psychological outcomes for both the resident and their carers have been linked with care home admission (Gaugler, Pot \& Zarit, 2007; Schulz et al., 2004; Sury, Burns, \& Brodaty, 2013). Some caregivers report reduced stress after placement of a relative. Others find their anxiety continues when their carer role changes and is not fully relinquished to the care home staff. New residents can experience improvements in both mood and short-term physical health. However, many who are living with dementia experience depression, agitation, worse physical health and social withdrawal (Sury et al., 2013). They never adjust to placement or adjustment is complex and linked to cognitive and behavioural decline (Kydd, 2001; Wilson, 2008. Furthermore, these problems have been associated with poorer quality of life for residents (Beerens, Zwakhalen, Verbeek, Ruwaard, \& Hamers, 2013).

Based on personal construct and transition process theories, poor adjustment may be due to a resident's perception of there being no benefit to living in a care home or perceived incongruence between their new residence and their personal goals, values and sense of identity (Ellis, 2010). People with dementia adapt by placing emphasis on settling in, fitting in and finding meaning to integrate the relocation within their overall life history and selfidentity (Aminzadeh, Molnar, Dalziel, \& Garcia, 2013). 
Factors that influence transition outcomes for people with dementia after placement in a care home were identified in a systematic literature review (Sury et al., 2013). It highlighted that successful adjustment is facilitated by a person-centred approach comprising of orientation procedures, buddy systems and input into decision-making. Highly responsive care environments also prevent adverse reactions to relocation (Aminzadeh et al., 2013). Influential factors, negative symptoms associated with poor adjustment and predictors of adjustment have been consistent with non-dementia specific studies (Brownie, Horstmanshof, \& Garbutt, 2014; Lee, 2010).

Evidenced-based interventions to support healthy adjustment have not been developed, with only one qualitative study in this area published since 2011. This involved community psychiatric nurse visits to care home staff, family and relocated people with dementia, to provide, advice on behavioural management and to support family caregivers (Van Mierlo et al., 2015). The researchers concluded that six weeks post resident placement was too late to meet staff requirements.

However, the approach was primarily focused on continuity of care, staff need and was knowledge oriented. It did not address or evaluate resident factors, or produce a manual. There remains great need for interventions and research that promote successful adjustment for new care home residents with dementia, directly with and for new residents that can be delivered by staff and can be widely implemented.

The aim of this study was to develop and validate the feasibility of delivering SettleIN; an intervention focussed on promoting healthy adjustment in the initial stage of residency, for people with dementia following placement into a care home. Using a mixed methods within-participant design, it was hypothesised that SettleIN would (1) be acceptable to a wide range of relevant stakeholders, (2) be feasible for care home staff to implement and deliver, and (3) improve mood and quality of life for people with dementia. The intervention 
development, consultation phase and qualitative study results are reported here. A brief overview of quantitative methodology and outcome are included.

\section{Method}

A two-phase design based on the Medical Research Council's framework for the development of complex interventions was used to develop and validate the feasibility of the SettleIN intervention (Moore et al., 2015).

\section{Phase I: Intervention Development}

The SettleIN intervention detailed a four-week programme with a three-part framework which could be delivered over six weeks depending on staff lead shift pattern and availability. The first part, Needs Assessment, aimed to facilitate resident participation. Staff were guided to; enquire about the resident's current perspective about living in the care home, review the resident's physical health needs and use a high-level description of each healthy adjustment module to select the most relevant modules for the resident.

The core of the programme named Healthy Adjustment detailed four one-page modules named Orientation, Lifestyle, Family \& Friends, and Identity which were created by grouping the 15 positive and negative factors known to influence adjustment (Sury et al., 2013) according to relatedness and adjustment theory (Ellis, 2010). For example, the positive influential factor of devising 'This is Your Life' book was grouped with the negative influential factor of cultural dissonance in the form of language and/or other cultural issues under the module Identity. Each module guided staff to complete up to six activities (taking between 10-30 minutes, designed to promote the positive factors and mitigate the negative factors) with the resident (and families if they desired) over the course of four weeks. Continuing the example above and in line with adjustment theory (Ellis, 2010), to help the 
resident retain a sense of self and congruence between their location, values and identity, activities in the Identity module guided staff to undertake the 'This is Your Life' exercise, ask the resident about their values, religious preferences, important dates and ensure their related needs were met such as having their prayer mat. See Table 1 [Table 1 near here] for other module example activities. The Healthy Adjustment section also;

- began with a module planner and programme overview, a list containing each module, activity and how often and when the activity was to be completed enabling staff to track progress,

- contained templates for staff to capture activity outcomes e.g. a specific sheet for noting a resident's spiritual beliefs/needs, another for food likes/dislikes,

- guided staff to record notes in specifically designed templates about the resident response to each activity e.g. an example included 'smiled a lot, told story about his Dad'

- provided staff with example questions to ask residents when undertaking activities within the modules.

The third part of the framework, Future Planning, involved gaining an up to date perspective from the resident about what they think about living in the care home and guiding staff to consider how any adjustment progress made might be maintained.

Two colourful manuals were created (i) a manual for care home managers that included the theoretical framework and values of the programme and (ii) a workbook for staff that contained the instructions and recording elements of the programme.

\section{Stakeholder consultation and results.}

Forty-seven clinical, academic, care home staff, healthcare and family stakeholders were recruited for consultation through lead researcher contacts (Table 2) [Table 2 near here]. 
Two large groups; (i) a London based community memory service team (n=12) and (ii) a non-London based Alzheimer's Society carer group $(n=14)$ were involved along with nine individual interviews (six face to face, three email) and 11 small groups with up to four stakeholders each. Insight was sought into the acceptability of the activities and whether the materials were easy to understand and feasible. A programme overview was provided, stakeholders were invited to appraise the documentation and an interactive semi-structured discussion followed based on a pre-developed discussion guide. Stakeholders were advised of the intended use of feedback collected, that it would be anonymised and were given opportunity to withdraw themselves and their feedback. Digital or written notes recorded the consultations that averaged 60 minutes in duration.

Thirty-seven stakeholders consented to completing a questionnaire that comprised of open and closed queries about the intervention. Examples include (i) 'How feasible do you think it will be to deliver SettleIN? Completely unfeasible; Slightly feasible; Neither feasible or Unfeasible; Somewhat feasible; Completely feasible', (ii) 'Overall, how does SettleIN compare to Care as Usual?', and (iii) What do you like least about the SettleIN programme?

Most stakeholders indicated that SettleIN was completely appropriate (68\%), realistic (79\%) and anticipated that delivery of SettleIN within care homes would be feasible $(97 \%$, $\mathrm{n}=28$ ). Thematic analysis of consultation records and responses to open questions in the questionnaire (see Supplemental Table S1 and S2) [Table S1 and S2 near here] indicated that stakeholders found SettleIN to be a helpful, well-structured, comprehensive tool which was likely to aid staff to understand and better meet resident needs. Several mentioned that SettleIN should be part of standard practice and training. While some stakeholders thought SettleIN would be easy to use, others warned of likely barriers to implementation including limited resident communication skills, high staff turnover and a 'tick-box' attitude. 


\section{Intervention refinement after consultation.}

Following stakeholder feedback the original SettleIN documentation was divided into two manuals as described above. The module planner (to help staff monitor progress) and pictures of faces (to enable ease of answering for residents indicating their perspective on living in the home) were additions following stakeholder feedback.

\section{Phase II: Feasibility Study}

\section{Setting}

Fifteen UK care homes were identified by: seeking interest from manager attendees at a care home research event, supervisor networks and cold calling homes local to the lead researcher, found through the 2015 UK Care Quality Commission (CQC) care home directory. Inclusion criteria involved care homes that accepted residents with dementia and had attained CQC service standards of good or above. Nine managers agreed to a meeting with the lead researcher to gain further information and of these, six, from homes in three UK counties, accepted the invitation to participate. One home had a CQC rating of outstanding and five were rated good. Each home offered between 30 and 90 beds and was privately owned. After initial staff training had taken place, one home (rated 'good') was excluded; they no longer had eligible residents as their bed status changed to 'for respite'.

\section{Recruitment}

Fifteen of 16 new residents and families identified by care home managers expressed an interest in the study. The lead researcher explained the study in more detail, confirmed eligibility and, where relevant, gained consent for participation. Residents were eligible to participate if they;

- were aged 65 years and older, 
- had been in permanent care home residence for less than four weeks,

- had a diagnosis of dementia as defined by the Diagnostic and Statistical Manual of Mental Disorders (5 ${ }^{\text {th }}$ ed.; DSM-5; American Psychiatric Association, 2013)

- had a Functional Assessment Staging Test (FAST; Reisberg, 1987) score of between mild (2) and moderately severe (6e).

- could understand and communicate in English.

One resident did not meet eligibility criteria and another was withdrawn by the family due to health deterioration.

\section{Participants}

Thirteen residents from five care homes were included, all aged 70 years old and above. Family involvement in the research varied from solely granting consent to assisting with intervention activities and included seven daughters, three sons, one spouse, one daughter-in-law and one non-related proxy.

Twenty-four staff were recruited and trained in using SettleIN. Staff participants were proposed by care home managers or team leaders from all six care homes and included resident participants' key workers. Staff who attended training were given a certificate and those that led a SettleIN programme were given a small gift voucher following study completion.

\section{Ethical Approval}

Ethical approval was granted by the Health Research Authority Research Ethics Committee Camden and Kings Cross and University College London Joint Research Office (15/LO/0611). Written consent was obtained from all care home managers, staff members 
and family or proxy representatives of each resident participating in the research. Data obtained was made anonymous and identifying information stored separately.

\section{Training}

Training varied in nature due to staff availability and the care home setting. The threehour planned session was often condensed to one and a half hours, took place in a communal area or separate room and included between one and three participants at a time. The lead researcher explained the overarching aims and values of the programme before working through the SettleIN workbook with staff participants. Didactic teaching, role plays and question and answer components were applied.

\section{Intervention and Procedure}

Staff applied the intervention described in Phase I and completed the SettleIN user guide and workbook with the resident (and where involved, the family) undertaking one or more activities over the course of a shift. Staff used the planner and workbook templates to record which activities had been completed, information outcomes gathered during activities, observable resident responses to undertaking activities and resident perspectives on living in the home. The lead researcher was available for telephone support throughout the programme and contacted the care home weekly to check progress and assist.

\section{Measures}

The Functional Assessment Staging Test (FAST, Reisberg, 1987) was chosen to measure the stage of dementia relating to functional deterioration in participants and the score was predominantly ascertained though an interview with the staff. FAST is an empirically supported ordinal scale depicting seven stages ranging from normal function to severe 
dementia (Sclan \& Reisberg, 1992). Stages six and seven are divided into subscales and generate 16 possible ratings. Moderately severe dementia is indicated at stage $6 \mathrm{a}$ and above.

The Index of Relocation Adjustment scale (IRA; Prager, 1986), The Quality of Life in Alzheimer's Disease (QOL-AD, Logsdon, Gibbons, McCurry, \& Teri, 1999) and The Cornell Scale for Depression in Dementia (CSDD, Alexopoulos, Abrams, Young, \& Shamoian, 1988) were selected for their parametric credentials and specifically for the latter two measures, their extensive use when working with people with dementia.

\section{Data collection}

The FAST was collected with staff or family at baseline to check that residents met the inclusion criteria. The lead researcher also collected the participants' demographic details from care records and all other baseline measures from the staff lead, family carer and the resident (where possible).

Other members of the research team collected the time two and three measures, copies of the workbook and recorded field reports of staff feedback during data collection. They also conducted semi structured interviews with staff intervention leads post programme delivery based on a pre-developed guide. Topics included likes and dislikes of using SettleIN, aspects most difficult/easiest to deliver and why, how SettleIN compared to Care as Usual, SettleIN language, detail and design (clarity of instructions, programme length), changes needed to improve practicality, impact on residents and staff. Transcripts of the staff interviews, staff notes of resident responses to activities within SettleIN workbooks, and researcher field reports formed the sources of qualitative data. 
Qualitative: Thematic analysis.

The qualitative data sources were made anonymous and imported into NVivo, version 11 (QSR International, 2015). Both the data-driven inductive approach of Boyatzis (1998) and the deductive a priori template of codes approach outlined by Crabtree and Miller (1999) were applied to interpret and understand the data. Two a priori codes of feasibility and resident outcomes were created according to research questions and hypotheses. The data was systematically coded over three rounds and considered for relevance to an a priori code or creation of a new code. This was followed by axial and selective coding. Constant comparison of each case and dataset was applied for coding consistency. Data contrasting with any themes and codes were identified. The lead researcher held a critical realism philosophy. To assess reliability and validity of analysis and results, two independent research volunteers (one experienced and one new to qualitative research) coded two randomly selected staff interviews and one researcher field note. Participating care home managers were invited to appraise a summary of the qualitative findings.

\section{Results}

\section{Phase II: Feasibility Study}

The eight female and five male residents included in the study ranged in age from 70 to 97 (mean, 87.9; sd, 7.2). Eleven were from White-British backgrounds (85\%), eight were widowed (62\%) and two were married.

Of the 24 staff, two withdrew from the study after SettleIN training had been completed. The remaining 22 staff were predominantly female (90\%), ranged in age from 18 to 64 years, averaged nine years of providing dementia care and many used English as a second or third language (45\%). Additional resident and staff demographic characteristics 
are presented in Table 3 [Table 3 near here] below. Of the 22 trained staff, 15 were assigned to lead or co-lead delivery of SettleIN for a new resident while one was a deputy manager who intended to provide support to staff leads. The remaining six staff awaited assignments to a dyad following new resident recruitment.

While recognised resident measures with accrued reliability and validity were used in this study at baseline, time two (within a week of intervention completion) and time three (four weeks later), their specifics and quantitative results are not provided here; high attrition and the low sample size rendered planned mixed model analyses unusable. For completeness, the flow of intervention measure collection is presented in Figure 1 and show baseline $(n=13)$, time two $(n=5)$ and time three $(n=4)$ data impacted by high drop-out rates due to resident hospitalisation or death $(n=4)$ and non-delivery by staff $(n=4)$.

One measure, The Index of Relocation Adjustment scale (IRA; Prager, 1986) that was selected as an explorative, general measure of adjustment and referred to the ability of older adults to cope with different demands and to stabilise as members of a residential home community (Lee, Woo, \& MacKenzie, 2002) was well received by residents. Of the twelve attempts made to collect IRA at baseline, nine were acquired and only one had missing items. These measure uptake results are useful for future adjustment studies as while support for IRA's reliability and construct validity have been accrued (Bekhet \& Zauszniewski, 2014) its psychometric properties have yet to be evaluated within a dementia population.

\section{Qualitative Results}

Six post intervention staff interviews, four sets of SettleIN workbook field notes, and 11 researcher field reports informed the qualitative results. Overall, themes (derived from 73 open, ten axial and four selective codes) indicated that when SettleIN was implemented it was better than care-as-usual and easily integrated into care for some, though not all 
residents. However, full delivery of all intervention components was frequently impeded by dependency on family involvement. When SettleIN was not implemented, staff reported barriers of lack of time, high workload, and low staff to resident ratios. While staff found SettleIN to be beneficial to themselves, they also reported contrasting themes of positive change and no benefit for resident quality of life and mood. Proposed intervention enhancements included further programme simplification and reduced dependency on family. See Supplemental Table S3. [Table S3 near here] Theme details including quotation evidence are reported below according to hypotheses topics of (i) feasibility and (ii) outcomes. This is followed by an additional theme (iii) improvements and results from data validation.

Independent rating identified a small number of instances where data had not been coded or only one code had been applied where two codes may be relevant. No additional codes were proposed and subsequent checks for consistency did not change axial codes or overall themes.

Feasibility and barriers when implementation was undertaken.

Staff who led the programme delivery found the SettleIN workbook to be; easy to understand and use, have clear language and detail a well explained programme. Staff spoke of the overall programme duration being practical and four staff found the proposed length of time for each activity to be either helpful or accurate. SettleIN was incorporated into normal care activities. Four staff indicated that the SettleIN was better than care-as-usual due to; the peace of mind and continuity of life SettleIN enabled for residents, the insight facilitated for staff into a resident's pre-placement life and, aid with getting to know a new resident rapidly. 
'...It's quite self-explanatory really... once you have got that module in front of you then it's got it all there, what you have to do... Completely feasible.' (Health Care Assistant (HCA): Interview 1)

'I would say...we would have found out what everybody found out from him...eventually... [SettleIN] just made it a lot quicker.' (Senior HCA: Interview 4)

Staff reported that intervention delivery was halted or incomplete when a resident's health deteriorated, they were hospitalised or died. Staff highlighted the main barrier to fully completing a module activity (and a key source of frustration) was waiting for family to provide specific pieces of information, a memorable item or a particular resource that the intervention component required.

'...they weren't so helpful ...[The family said] we will bring it next week, came next week, nothing..' (HCA: Interview 2)

Three staff experienced difficulties when attempting to deliver SettleIN. One resident's minimal communication style meant it was hard to engage with them and determine if the activity was enjoyed. One reluctant resident was suspicious of SettleIN activities. Staff concluded that SettleIN may not be suitable for all residents despite its utility.

‘... it's a shame we couldn't have done this programme with [Resident's name]... it's a very useful thing with some of the residents...I don't think [SettleIN is] inappropriate 
at all, but you've obviously got to make a sort of decision ...to whether or not that person is suitable for it.' (SHCA: Interview 3)

Feasibility and barriers when implementation did not take place.

Staff from two care homes reported they did not start or commit to SettleIN due to a lack of time, high workload, and low staff to resident ratios.

'I don't have that much time to deal with these things...' (HCA: Interview 6)

'Care staff...mentioned their high workload...there were only four [staff] to care for many residents (32)...[staff] ...both reiterated that it was a lack of time that prevented them from completing the SettleIN modules.' (Researcher 4 field report)

Staff benefits yet resident outcome reports were contradictory.

Staff reported at least one positive change for each of the residents who received SettleIN. Staff described residents' increased familiarity with people and their new surroundings. Improvements in resident; mood, increased trust and development of connections with others, reduction in unwanted resident behaviour and an increase in engagement with care home life were also reported.

'SettleIN... helped a lot... I know that now he's a late riser...he actually remembered my name...it actually made him settle in ...it was like a challenge for him... when I used to say... 'Can you show me to the lounge?'. At first it was like going for a long mission, around the whole home...in the end .. he knew his way...[and] when he first 
came here he was quite aggressive with other residents ...two or three times a day...I haven't seen him lash out properly in ages' (HCA: Interview 2)

In contrast, three of the same staff perceived that the identical residents had not benefited from the programme and one highlighted the difficulty staff found in communicating with a resident and discerning differences in responses.

'I think with her it just didn't work.' (SHCA: Interview 3)

'I don't think this resident benefited from it'. (SHCA: Interview 4)

Staff experienced an array of benefits including; a sense of achievement, increased confidence, learning and development, knowledge sharing between staff and development of bonds with residents. One suggested all staff should use SettleIN to develop staff understanding of adjustment.

'We achieved something... it was like I achieved something with him, that I might have got through to him, like just knew a little bit more about him.' (HCA: Interview 5)

\section{Improvements to SettleIN.}

Staff proposed that SettleIN might be improved by; simplifying the core offering, reducing the frequency of repetition and intensity of some of the questions, and by making minor amendments to the activity schedule, terminology and recording sheets. The difficulties reported in working with some families highlighted the need to remove dependency on family for module delivery. 
'[The planner] should just be a block for however many units... [activities] should be completed in that week, instead of the days.' (HCA: Interview 2)

Validation of Qualitative Results.

Two of the five care home managers responded to the invitation to appraise the themes found. They confirmed that the results were representative of their experience. No counter theme examples were identified. One manager stated,

'There was no difficulty in implementing the programme at all...I think that family carers would experience higher levels of satisfaction with care after SettleIN'.

(Manager, Care home with CQC rating of Outstanding) 


\section{Discussion}

\section{Summary of findings}

This study hypothesised that SettleIN would (1) be acceptable to a wide range of relevant stakeholders, (2) be feasible for care home staff to implement and deliver, and (3) improve mood and quality of life for people with dementia. In a consultation phase, experts responded positively to the underpinning theory, content, clarity of the programme and accompanying documentation, providing some support for hypothesis (1). During a feasibility study, staff found SettleIN to be easily integrated into care, better than care-asusual, and of benefit to staff. However, significant barriers to implementation and comprehensive delivery were identified; lack of staff time, unsuccessful dependency on family involvement and lack of suitability for all new residents with dementia. Hypothesis (2) was therefore only partially supported. While mixed model analysis was undertaken, the high attrition (62\% at time two) and low uptake meant there was too small a sample for the validated assessment measures and quantitative analysis to substantiate the positive quality of life and mood outcomes for residents. These results were therefore not reported and hypothesis (3) was not supported. The captured concerns and ideas for improvement provided understanding of likely reasons for non-implementation and incomplete delivery.

\section{Feasibility of implementing and delivering SettleIN}

Despite programme delivery in multiple homes and some managers confirming that implementing SettleIN was feasible, other homes, where staff described being stretched, task focussed and working with low staff-to-resident ratios, struggled to begin implementation. These are common challenges in care home research and emphasise why interventions need to be very easily incorporated into usual care practice (Lawrence, Fossey, Ballard, Moniz Cook, \& Murray, 2012; Luff, Ferreira, \& Meyer, 2011). 
SettleIN was not started on four occasions, with unanticipated scenarios such as unplanned staff leave being contributing factors. Family involvement is linked with positive outcomes for residents (Castro-Monteiro et al., 2016) however in this study, when families did not engage, staff were unable to fully deliver SettleIN.

\section{Potential improvements}

In this study, eligible resident participants were within their first four weeks of care home placement. This may be too late to prevent decline in physical health and hospitalisation; key contributors to high attrition in this study and negative outcomes of nonadjustment (Sury et al, 2013). Therefore, SettleIN needs to be started earlier, from day one of residency and be fully integrated into the relocation process to support healthy adjustment. Furthermore, to optimise the benefits of using SettleIN, including staff reported increased; confidence, knowledge of adjustment and bonds with residents, all staff need to be trained in SettleIN and new staff need to have access to SettleIN training.

Uptake and support for SettleIN implementation by management and staff may be increased by improving training about how SettleIN can be fully integrated into care as usual without adding any additional task time. Provision of case studies from this pilot, highlighting the positive change for residents as identified by staff (and in one case the reduction in unwanted behaviour which can also be demanding and time consuming for staff) may also increase willingness to implement SettleIN.

SettleIN would be enhanced by simplifying the core offering with concentration on the healthy adjustment section. Reducing the number of activities within each module and reducing the frequency in which the activities are undertaken may also simplify SettleIN and increase feasibility. Removing dependency on families would aid intervention delivery without preventing families from being involved if they wished to do so. 
A review of the question prompts provided and how they are utilised would ensure they are not experienced as intense. While each question such as 'what time do you usually get up?', 'what is the first thing you do?', or 'do you get dressed first or have a cup of tea or coffee first?' may be appropriate for the resident, if too many questions are asked within one activity, the experience may become overwhelming. The overall length of the programme could be reviewed. Amendments made to the activity schedule to display weekly rather than daily units would be better aligned to staff shift work. Minor alterations to terminology such as replacing 'buddy' with 'friend' and providing more space within recording sheets would also address staff feedback.

\section{Methodology Strengths and Limitations}

Consultation with 47 relevant experts including family representatives and the underpinning of SettleIN in theory and evidence were strengths of the intervention development and led to a high level of confidence in SettleIN prior to trial. Response bias was avoided by the use of independent data collectors from those used at baseline. A mixed method design avoided the suggested benefits of the intervention being overlooked and reliability and validity checks were undertaken. Valuable insight has been provided into the large target sample size required for a pilot of SettleIN to meet statistical power requirements with over 80 participants needed if a similar retention rate of $30 \%$ was assumed.

This study met with many of the attrition related obstacles that are common to conducting research with people with dementia in care home settings including participant death and hospitalisation, low staffing levels and inconsistent managerial support (Luff et al.,

2011). Reduced training time due to lack of staff availability may have impacted implementation; SettleIN was not started in homes where the least amount of training took place. Hospitalisation or death were the predominant cause of attrition and was out of control 
of the researchers. The addition of a control group would add strength to this study's design and help to distinguish between natural and intervention related adjustment.

\section{Clinical implications.}

This study confirms how difficult some care homes find implementing new interventions, even those they and other experts perceive as worthwhile. Currently there is no best practice guideline for care homes about how to provide assistance with healthy adjustment to a person with dementia in their first few weeks of care home residency. SettleIN provides a first step towards development of best practice in response to the growing numbers of people with dementia who are likely to reside in care homes over upcoming years.

\section{Research implications.}

A well powered pilot of an enhanced version of SettleIN is needed to establish if it is feasible and results in positive outcomes for resident quality of life and mood. A firmer commitment from staff and higher numbers at baseline (to allow for attrition) would be necessary. Reducing the broad FAST level inclusion criterion may be worthwhile to decrease the likelihood of resident hospitalisation or death impacting a future pilot though this can never be fully mitigated.

\section{Conclusion}

Despite its limitations, this is the first known study of an intervention to support healthy adjustment in people with dementia in the early period of residency following placement in a care home. A few core feasibility concerns and high attrition mean intervention 
improvements are required. A second pilot is needed to investigate the impact of an enhanced SettleIN on new resident mood and quality of life. 


\section{Acknowledgements:}

We thank the care staff, managers, and residents who took part in this study. We also thank Stella Spanos for assistance with the consultation phase and Emily Kitson, Christopher Albertyn, Anne Sakoane and Maria Gkini for their invaluable help with data collection.

\section{Declaration of conflicting interest:}

The authors declare that there is no conflict of interest. 


\section{References}

Alexopoulos, G. S., Abrams, R. C., Young, R. C., \& Shamoian, C. A. (1988). Cornell scale for depression in dementia. Biological Psychiatry, 23(3), 271-284.

Alzheimer's Disease International (ADI). (2015). World Alzheimer Report 2015. London: Alzheimer's Disease International.

Aminzadeh, F., Molnar, F. J., Dalziel, W. B., \& Garcia, L. J. (2013). An exploration of adjustment needs and efforts of persons with dementia after relocation to a residential care facility. Journal of Housing for the Elderly, 27(1-2), 221-240.

Beerens, H. C., Zwakhalen, S. M., Verbeek, H, Ruwaard., D., \& Hamers, J. P. (2013). Factors associated with quality of life of people with dementia in long-term care facilities: a systematic review. International Journal of Nursing Studies, 50(9), 12591270.

Bekhet, A. K., \& Zauszniewski, J. A. (2014). Psychometric properties of the index of relocation adjustment. Journal of Applied Gerontology, 33(4), 437-455.

Boyatzis, R. E. (1998). Transforming qualitative information: Thematic analysis and code development. Sage.

Brownie, S., Horstmanshof, L., \& Garbutt, R. (2014). Factors that impact residents' transition and psychological adjustment to long-term care: a systematic literature review. International Journal of Nursing Studies, 51(12), 1654-1666. 
Castro-Monteiro, E., Alhayek-Aí, M., Diaz-Redondo, A., Ayala, A., Rodriguez-Blazquez, C., Rojo-Perez, F., ... Forjaz, M. J. (2016). Quality of life of institutionalized older adults by dementia severity. International Psychogeriatrics, 28(1), 83-92.

Comas-Herrera, A., Northey, S., Wittenberg, R., Knapp, M., Bhattacharyya, S., \& Burns, A. (2011). Future costs of dementia-related long-term care: Exploring future scenarios. International Psychogeriatrics, 23(1), 20-30.

Crabtree, B. F., \& Miller, W. L. (Eds.). (1999). Doing qualitative research. Sage publications.

Ellis, J. M. (2010). Psychological transition into a residential care facility: Older people's experiences. Journal of Advance Nursing, 66(5), 1159-1168.

Gaugler, J., Pot. A., \& Zarit, S. (2007). Long-term adaptation to institutionalization in dementia caregivers. The Gerontologist, 47(6), 730-740.

Hodgson, N., Freedman, V. A., Granger, D. A., \& Erno, A. (2004). Biobehavioral correlates of relocation in the frail elderly: Salivary cortisol, affect, and cognitive function. Journal of the American Geriatrics Society, 52(11), 1856-1862.

Kydd, P. (2001). Using music therapy to help a client with Alzheimer's disease adapt to longterm care. American Journal of Alzheimer's Disease and Other Dementias, 16(2), 103-108. 
Lawrence, V., Fossey, J., Ballard, C., Moniz Cook, E., \& Murray, J. (2012). Improving quality of life for people with dementia in care homes: Making psychosocial interventions work. British Journal of Psychiatry, 201(5), 344-351.

Lee, D. T., Woo, J., \& MacKenzie, A. E. (2002). A review of older people's experiences with residential care placement. Journal of Advanced Nursing, 37(1), 19-27.

Lee, G. E. (2010). Predictors of adjustment to nursing home life of elderly residents: A crosssectional survey. International Journal of Nursing Studies, 47(8), 957-964.

Logsdon, R. G., Gibbons, L. E., McCurry, S. M., \& Teri, L. (1999). Quality of life in Alzheimer's disease: Patient and caregiver reports. Journal of Mental Health \& Aging, $5(1), 21-32$.

Luff, R., Ferreira, Z., \& Meyer, J. (2011). Care Homes SSCR Methods Review 8. London, UK: NIHR School for Social Care Research. Retrieved from http://sscr.nihr.ac.uk/PDF/MR/MR8.pdf

Moore, G. F., Audrey, S., Barker, M., Bond, L., Bonell, C., Hardeman, W., ... Baird, J. (2015). Process evaluation of complex interventions: Medical Research Council guidance. British Medical Journal, 350, h1258.

Prager, E. (1986). Components of personal adjustment of long distance elderly movers. The Gerontologist, 26(6), 676-680. 
Prince, M., Bryce, R., Albanese, E., Wimo, A., Ribeiro, W., \& Ferri, C. P. (2013). The global prevalence of dementia: a systematic review and metaanalysis. Alzheimer's \& Dementia, 9(1), 63-75.

QSR International. (2015). NVivo qualitative data analysis software (Version 11). Software. Doncaster, VIC, Australia: QSR International Pty Ltd.

Reisberg, B. (1987). Functional assessment staging (FAST). Psychopharmacology Bulletin, 24(4), 653-659.

Sclan, S. G., \& Reisberg, B. (1992). Functional assessment staging (FAST) in Alzheimer's disease: Reliability, validity, and ordinality. International Psychogeriatrics, 4(Suppl. 1), 55-69.

Schulz, R., Belle, S. H., Czaja, S. J., McGinnis, K. A., Stevens, A., \& Zhang, S. (2004). Long-term care placement of dementia patients and caregiver health and well-being. JAMA: The Journal of the American Medical Association, 292(8), 961-967.

Smith, A. E., \& Crome, P. (2000). Relocation mosaic: A review of 40 years of resettlement literature. Reviews in Clinical Gerontology, 10(1), 81-95.

Sury, L., Burns, K., \& Brodaty, H. (2013). Moving in: Adjustment of people living with dementia going into a nursing home and their families. International Psychogeriatrics, 25(6), 867-876. 
Van Mierlo, L. D., Bootsma-Van der Wiel, A., Meiland, F. J., Van Hout, H. P. J., Stek, M. L., \& Dröes, R. M. (2015). Tailored mental health care after nursing home admission: improving transfers of people with dementia with behavioral problems. An explorative study. Aging and Mental Health, 19(10), 902-911.

Wilson, C. B. (2008). Using relationships to develop practice that values the contribution of older people, families and staff. International Journal of Older People Nursing, 3(4), 274-277. 


\section{Figure/Table Legends}

\section{Figures}

Figure 1. Flow of intervention measure collection and attrition

\section{Tables}

Title:

Table 1 Examples of one aim and an activity from each of the four SettleIN modules.

Legend:

Note. Frequency refers to different days unless stated. Minutes refers to per attempt.

Title:

Table 2 Demographic characteristics of stakeholder consultants

Legend:

Note. Percentages are rounded so may not total to exactly $100 \%$

Title:

Table 3 Characteristics of resident and staff participants

Legend:

Note. Percentages are rounded so may not total to exactly $100 \%$. No Fast $6 \mathrm{a}-\mathrm{b}$ found. 